\title{
Semantic Web Public Policy Challenges: Privacy, Provenance, Property and Personhood
}

\author{
Daniel J. Weitzner \\ Co-director, MIT Decentralized Information Group (DIG), \\ Technology and Society Domain Lead, World Wide Web Consortium (W3C) \\ http://www.w3.org/People/Weitzner.html
}

\begin{abstract}
The growing inferencing and knowledge linking power of the Semantic Web will, we all hope, make the world a better place: enrich democratic discourse, support more rapid scientific discovery, enable new forms of personal communication and culture, and generally enhance critical analysis of information. However, with this greater inferencing power comes daunting social and public policy questions that must be faced as first class technical design challenges, not just as issues to be resolved in courts and legislatures. How will we maintain fundamental privacy values in the face of inferencing and searching power that can systematically uncover sensitive facts about us even has we try to keep such data secret? Today's Web has enabled a departure from traditional editorial control and historically-trusted information sources. Will attention to provenance on the Semantic Web enable us to develop new mechanisms for assessing the reliability of information? What new challenges to already frayed intellectual property regimes will the Semantic Web bring? Finally, how will we assert and represent personal identity on the Semantic Web? At this early stage of the development of the Semantic Web, it's hard enough to have problems in focus, much less solutions. However, we believe that transparent reasoning and accountability mechanisms will play a critical role in enabling systems and services built on the Semantic Web to be more responsive to social and policy needs.
\end{abstract}

